\title{
过渡金属催化有机腈和叠氮酸钠反应机理的研究
}

\author{
黄荣谊* 沈琼张超张少勇徐衡* \\ (安庆师范大学化学化工学院 功能配合物安徽省重点实验室 安庆 246011)
}

\begin{abstract}
摘要 过渡金属催化有机腈和叠氮酸钠反应机理的研究一直是一个具有挑战性的课题. 本工作组合理论和实验方法, 对其反应机理作了深入的探讨. 基于对两种类型中间体 $\left(\mathrm{H}_{2} \mathrm{O}\right)_{3} \mathrm{M} \cdots \mathrm{N}_{3}-$ 和 $\left(\mathrm{H}_{2} \mathrm{O}\right)_{3} \mathrm{M} \cdots \mathrm{NCCH}_{3}$ 稳定性的理论分析和中间体 $\mathrm{Cd}_{2}\left(\mu_{3}-\mathrm{N}_{3}\right)\left(\mu_{3}-\mathrm{OH}\right)\left(\mu_{5}-\mathrm{CHDA}\right)(\mathbf{1})$ 和 $\mathrm{Cd}\left(\mu_{2}-\mathrm{N}_{3}\right)\left(\mu_{3}-\mathrm{IBA}\right)(2)\left(\mathrm{H}_{2} \mathrm{CHDA}\right.$ 为 1,3-环已二酸, HIBA 为 4'-(咪唑-1-基)苯甲酸)的成 功捕获，首次从实验和理论上揭示了过渡金属离子在反应中起活化叠氮酸根的作用. 此外，基于中间体 $\left(\mathrm{H}_{2} \mathrm{O}\right)_{3} \mathrm{M} \cdots \mathrm{N}_{3}{ }^{-}$ 和乙腈分子的静电势盆分析的结果，揭示了叠氮酸根未配位的端基 $\mathrm{N}$ 原子可以进攻氰基 $\mathrm{N}$ 原子发生加成反应，随后可 能发生环加成反应生成四唑, 在水分子的辅助下, 其加成物也可能发生类似于里特反应生成全氮阴离子. 该项研究把 过渡金属催化有机腈和叠氮酸钠反应引入全氮阴离子合成的新领域.
\end{abstract}

关键词 金属催化; 反应机理; 镉(II)配位聚合物; 量子化学计算; 全氮阴离子

\section{Studies on the Mechanism of the Transition Metal-Catalyzed Reaction of Organonitrile with Sodium Azide}

\author{
Huang, Rongyi* $\quad$ Shen, Qiong Zhang, Chao Zhang, Shaoyong $\quad \mathrm{Xu}$, Heng* \\ (Anhui Key Laboratory of Functional Coordination Compounds and School of Chemistry and Chemical Engineering, Anqing \\ Normal University, Anqing 246011)
}

\begin{abstract}
The study on the reaction mechanism of organonitrile and sodium azide catalyzed by transition metals has always been a challenging and controversial task. Due to the difficulty in capturing the reaction intermediates, there is still no direct evidence to uncover the nature of the reaction. In this paper, the reaction mechanism has been explored by using a combining theoretical and experimental method. Based on the theoretical analysis of the stability of two types of intermediates $\left(\mathrm{H}_{2} \mathrm{O}\right)_{3} \mathrm{M} \cdots \mathrm{N}_{3}{ }^{-}$and $\left(\mathrm{H}_{2} \mathrm{O}\right)_{3} \mathrm{M} \cdots \mathrm{NCCH}_{3}$ and the successful capture of two activated intermediates containing metal cadmium ions $\mathrm{Cd}_{2}\left(\mu_{3}-\mathrm{N}_{3}\right)\left(\mu_{3}-\mathrm{OH}\right)\left(\mu_{5}-\mathrm{CHDA}\right)(\mathbf{1})$ and $\mathrm{Cd}\left(\mu_{2}-\mathrm{N}_{3}\right)\left(\mu_{3}-\mathrm{IBA}\right)(2)\left(\mathrm{H}_{2} \mathrm{CHDA}=1,3\right.$-cycloadipic acid and HIBA=4-(imidazol-1-yl) benzoic acid), which were achieved under the hydrothermal conditions and characterized by single-crystal XRD analysis. For the first time, the experimental and theoretical results reveal that the transition metal ions activate the azide rather than the cyano group of nitriles. In addition, the results of both the electrostatic potential basins analysis of activated intermediates $\left(\mathrm{H}_{2} \mathrm{O}\right)_{3} \mathrm{M} \cdots \mathrm{N}_{3}{ }^{-}$and acetonitrile molecules obtained by the theoretical calculation and our recently reported experimental results reveal that the intermediates $\left(\mathrm{H}_{2} \mathrm{O}\right)_{3} \mathrm{M} \cdots \mathrm{N}_{3}{ }^{-}$can be used as electrophilic reagent. Its uncoordinated terminal $\mathrm{N}$ atom can attack the $\mathrm{N}$ atom of the cyano group of acetonitrile to undergo a nucleophilic addition reaction during the chemical reaction progress, and then it may undergo a [2+3] cycloaddition reaction to in-situ form tetrazole. Moreover, with the aid of water molecules, its adducts may also occur similar to the Ritter-like reaction to in-situ form polynitrogen anion. Our findings may open a novel field of the $i n-s i t u$ synthesis of polynitrogen compounds based on the transition metal-catalyzed reactions of organonitrile and azide.
\end{abstract}

Keywords metal catalysis; reaction mechanism; cadmium(II) polymer; quantum chemical calculation; polynitrogen anion

\section{1 引言}

随着配位化学的发展, 特别是 Sharpless 及其合作 者 $^{[1,2]}$ 报道了一种以水作溶剂的原位合成四唑的方法, 四唑配位化学取得了快速发展. 至今该反应机理还没有 被完全弄清楚，但金属催化下有机腈和叠氮酸钠发生原 位配体反应为四唑金属配合物的构筑提供了新的思路.
尤其是可以对四唑 5 位上的取代基进行功能化，使其构 筑的 MOFs 材料在气体储存、气体分离、催化、非线性 光学、分子光电功能材料以及含能材料等方面有着潜在 的应用 ${ }^{[3 \sim 14]}$.

近年来，在金属离子催化下有机腈和叠氮酸钠发生 原位配体反应合成四唑的反应机理的研究方面也受到 了关注 ${ }^{[15 ~ 18]}$. 目前, 研究的内容主要聚焦在下面的两个

\footnotetext{
*E-mail: aqhuangry@hotmail.com; aqxuhengg@163.com

Received March 24, 2020; published May 14, 2020.

Supporting information for this article is available free of charge via the Internet at http://sioc-journal.cn.

Project supported by the National Natural Science Foundation of China (No. 21975003) and the Program for Innovative Research Team in Anqing Normal University.

项目受国家自然科学基金(No. 21975003)和安庆师范大学科研创新团队建设计划资助.
} 
方面: (1)金属离子在反应中所起的作用, 是活化叠氮酸 根还是活化有机腈? (2)叠氮酸根和有机腈之间发生的 $[2+3]$ 环加成反应是两步分步反应还是一步协同反应, 或是两者可同时发生? 此外, 在反应机理研究方面, 虽 然获得了一些理论研究成果, 但是在实验上一直无法提 供有效的实验证据，困难在于反应中间体的捕获和表征 方面. 因此, 有机腈和叠氮酸根反应是一步反应还是两 步反应? 目前理论上提供的证据是不充分、不完备的. 尤其是在反应机理的理论研究方面, 主要关注的是 $[2+$ 3]环加成反应的机理部分, 而完全忽略了金属离子与有 机腈或叠氮酸根形成的反应中间体的特性和稳定性.

2003 年, 《美国化学会志》上报道了关于 $\mathrm{Zn}^{2+}$ 离子 在合成四唑反应中的作用的理论研究成果 ${ }^{[16]}$. 文章作 者分别计算了 $\mathrm{Zn}^{2+}$ 离子与乙腈和叠氮酸根形成的反应 中间体参与的一步协同反应的过渡态, 并获得了相应的 反应能垒. 基于反应能垒大小, 提出 $\mathrm{Zn}^{2+}$ 离子在反应中 的作用是活化氰基基团. 然而计算的两种类型的环加成 反应能垒差值较小, 仅在 $18.83 \sim 35.15 \mathrm{~kJ} / \mathrm{mol}$ 之间, 远 远地低于室温下分子的热运动产生的能量值 $(\approx 84.0$ $\mathrm{kJ} / \mathrm{mol}$ ). 作者单纯从反应能垒方面提出 $\mathrm{Zn}^{2+}$ 离子是活 化氰基基团的结论是不充分的. 另外, 作者既没有考虑 两种中间体在反应体系中的稳定性, 也没有考虑 $[2+3]$ 环加成反应为什么是一步协同反应, 而不是两步反应? 因此, 金属离子的作用是活化氰基基团还是活化叠氮酸 根仍需要提供可靠的实验和理论证据支持.

2015 年, 《德国应用化学》上发表了一篇关于过渡 金属离子在合成四唑反应中的作用的实验研究成果 ${ }^{[17]}$. 文章作者通过控制对苯二甲腈和叠氮酸钠的物质的量 比例 $(1: 2.5$ 和 $1: 1)$, 并利用水热结晶技术捕获到了金 属离子活化有机腈的中间体, 即在物质的量比为 $1: 1$ 时, 未参与形成四唑基团的对苯二甲腈的另一氧基与 $\mathrm{Cu}^{+}$离子配位生成的配合物. 基于此中间体作者提出金 属离子在反应中的作用是活化氰基. 然而, 这个中间体 是在氰基过量情况下获得的, 叠氮酸根已全部参与反应 形成四唑. 该结果并不能支持在反应体系中同时含对苯 二甲腈和叠氮酸根时, 金属离子只与氰基配位而不与叠 氮酸根配位起活化氰基的作用. 而从对苯二甲腈和叠氮 酸根本性来看, 叠氮酸根更易于和金属离子配位形成稳 定的配合物. 这与目前已报道的分别含芳腈配体和叠氮 酸根配体的配合物数目统计分析结果一致. 图 S1 为剑 桥晶体数据库中分别含有机芳腈和叠氮酸根配体的金 属配合物数目的统计结果. 从中可以看出, 含叠氮酸根 的金属配合物的数目比含芳腈配体的要多的多. 对于 $\mathrm{Cu}^{n+}$ 离子与叠氮酸根形成的配合物占两种配合物总数 的 $90.1 \%$. 从统计的角度来看, 金属离子也是更倾向于 与叠氮酸根配位, 其作用应该是活化叠氮酸根. 因此, 金属离子在反应中所起的作用仍然需要提供更多、更可 靠的实验和理论证据支持.
本文利用量子力学方法, 深入地研究了系列的过渡 金属离子在有机腈与叠氮酸根发生原位反应中的作用, 并利用水热合成技术，设计和成功捕获了两个金属 $\mathrm{Cd}^{2+}$ 离子活化叠氮酸根的中间体. 理论和实验结果均表 明在有机腈和叠氮化钠反应中过渡金属离子是起活化 叠氮酸根的作用. 此外, 在对中间体和有机腈的反应活 性的深入分析的基础上, 我们提出了新的见解, 即在过 渡金属离子催化下有机腈和叠氮酸根不仅可以发生 $[2$ +3 ]环加成反应合成四唑，也可以发生类里特反应合成 全氮阴离子. 这与我们近期报道的主族 $\mathrm{Pb}^{2+}$ 离子催化下 有机腈和叠氮酸根反应的结果一致 ${ }^{[19]}$.

\section{2 结果与讨论}

\section{1 几何构型与键级分析}

为了探讨在含金属离子、叠氮酸根和有机腈的反应 体系中，金属离子是活化叠氮酸根还是活化氰基，我们 采用 B 3LYP 密度泛函方法计算了 11 种含叠氮酸根的中 间体以及 11 种含乙腈的中间体(图 1). 表 1 列出了 22 种 中间体的 $\mathrm{M}-\mathrm{N}$ 配位键的键长以及 Mulliken 和 Mayer 键级. $\mathrm{M}-\mathrm{N}\left(\mathrm{N}_{3}{ }^{-}\right)$键的键长在 $0.1828 \sim 0.2063 \mathrm{~nm}$ 之间, $\mathrm{M}-\mathrm{N}\left(\mathrm{CH}_{3} \mathrm{CN}\right)$ 键的键长在 $0.1949 \sim 0.2202 \mathrm{~nm}$ 之间, 均 小于相应的两原子范德华半径之和，表明金属离子 $\mathrm{M}$ 和 $\mathrm{N}$ 原子间有成键效应. 另外, 对同种金属离子, 由于 $\mathrm{M}-\mathrm{N}\left(\mathrm{N}_{3}{ }^{-}\right)$键的键长比 $\mathrm{M}-\mathrm{N}\left(\mathrm{CH}_{3} \mathrm{CN}\right)$ 键的键长短, 键 级更大, 键的强度更强, 形成的中间体稳定性更高. 上 述结果表明在含金属离子、叠氮酸根和有机腈的反应体 系中, 叠氮酸根与金属离子形成的中间体比有机腈与金 属离子形成的中间体更稳定. 因此, 在反应体系中, 金 属离子可能是活化叠氮酸根而不是有机腈. 与文献 ${ }^{[15]}$ 报道的观点一致.

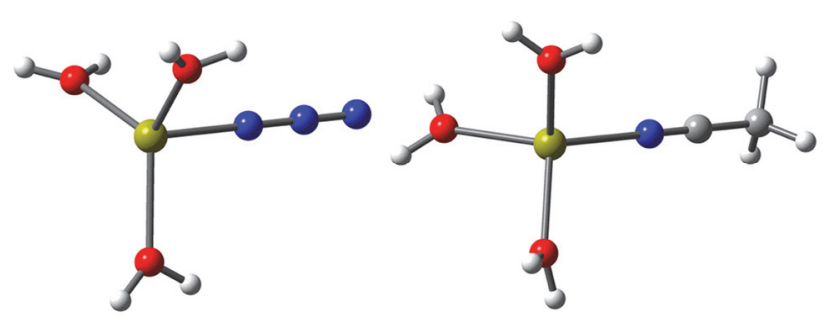

图 1 中间体 $\left(\mathrm{H}_{2} \mathrm{O}\right)_{3} \mathrm{M} \cdots \mathrm{N}_{3}{ }^{-}$和 $\left(\mathrm{H}_{2} \mathrm{O}\right)_{3} \mathrm{M} \cdots \mathrm{NCCH}_{3}$ 的分子结构模型 $(\mathrm{M}=\mathrm{Sc}, \mathrm{Ti}, \mathrm{V}, \mathrm{Cr}, \mathrm{Mn}, \mathrm{Fe}, \mathrm{Co}, \mathrm{Ni}, \mathrm{Cu}, \mathrm{Zn}$ 和 $\mathrm{Cd})$

Figure 1 The calculated structure models of the intermediates $\left(\mathrm{H}_{2} \mathrm{O}\right)_{3} \mathrm{M} \cdots \mathrm{N}_{3}{ }^{-}$and $\left(\mathrm{H}_{2} \mathrm{O}\right)_{3} \mathrm{M} \cdots \mathrm{NCCH}_{3}(\mathrm{M}=\mathrm{Sc}, \mathrm{Ti}, \mathrm{V}, \mathrm{Cr}, \mathrm{Mn}, \mathrm{Fe}, \mathrm{Co}$, $\mathrm{Ni}, \mathrm{Cu}, \mathrm{Zn}$ and $\mathrm{Cd}$ )

\section{$2.2 \mathrm{M}-\mathrm{N}$ 键的电子密度拓扑分析}

为了进一步理解两种类型中间体的 $\mathrm{M}-\mathrm{N}$ 配位键 的本性, 利用 AIM 理论对两种类型中间体进行了电子 密度拓扑分析. 图 2 给出了 $\mathrm{Zn}^{2+}$ 离子活化的两种中间体 的分子拓扑图. 从图中可以看出, $\mathrm{Zn}^{2+}$ 离子和四个配体 
表 1 中间体的 $\mathrm{M}-\mathrm{N}$ 键的键长和键级

Table 1 The bond lengths and bond orders of $\mathrm{M}-\mathrm{N}$ bonds of the intermediates

\begin{tabular}{|c|c|c|c|}
\hline Bonds & $R(\mathrm{M} \cdots \mathrm{N}) / \mathrm{nm}$ & Mulliken & Mayer \\
\hline $\mathrm{Sc} \cdot \cdots \mathrm{N}_{3}^{-}$ & 0.1967 & 0.2782 & 0.6510 \\
\hline $\mathrm{Sc} \cdots \mathrm{NCCH}_{3}$ & 0.2202 & 0.1409 & 0.2617 \\
\hline $\mathrm{Ti} \cdots \mathrm{N}_{3}{ }^{-}$ & 0.1947 & 0.2703 & 0.6005 \\
\hline $\mathrm{Ti} \cdots \mathrm{NCCH}_{3}$ & 0.2122 & 0.1782 & 0.3247 \\
\hline $\mathrm{V} \cdots \mathrm{N}_{3}^{-}$ & 0.1873 & 0.4798 & 0.8299 \\
\hline $\mathrm{V} \cdots \mathrm{NCCH}_{3}$ & 0.2026 & 0.2682 & 0.4452 \\
\hline $\mathrm{Cr} \cdots \mathrm{N}_{3}{ }^{-}$ & 0.1934 & 0.5487 & 0.7456 \\
\hline $\mathrm{Cr} \cdots \mathrm{NCCH}_{3}$ & 0.1979 & 0.2524 & 0.4443 \\
\hline $\mathrm{Mn} \cdots \mathrm{N}_{3}^{-}$ & 0.1873 & 0.4112 & 0.6364 \\
\hline $\mathrm{Mn} \cdots \mathrm{NCCH}_{3}$ & 0.2074 & 0.2838 & 0.3771 \\
\hline $\mathrm{Fe} \cdots \mathrm{N}_{3}^{-}$ & 0.1828 & 0.4542 & 0.6851 \\
\hline $\mathrm{Fe} \cdots \mathrm{NCCH}_{3}$ & 0.2019 & 0.3056 & 0.4026 \\
\hline $\mathrm{Co} \cdots \mathrm{N}_{3}{ }^{-}$ & 0.1854 & 0.6733 & 0.8224 \\
\hline $\mathrm{Co} \cdots \mathrm{NCCH}_{3}$ & 0.1981 & 0.3495 & 0.4468 \\
\hline $\mathrm{Ni} \cdots \mathrm{N}_{3}^{-}$ & 0.1837 & 0.7063 & 0.8608 \\
\hline $\mathrm{Ni} \cdots \mathrm{NCCH}_{3}$ & 0.1961 & 0.3870 & 0.4830 \\
\hline $\mathrm{Cu} \cdots \mathrm{N}_{3}^{-}$ & 0.1828 & 0.5960 & 0.7563 \\
\hline $\mathrm{Cu} \cdots \mathrm{NCCH}_{3}$ & 0.1949 & 0.3834 & 0.4949 \\
\hline $\mathrm{Zn} \cdots \mathrm{N}_{3}{ }^{-}$ & 0.1855 & 0.8348 & 0.9572 \\
\hline $\mathrm{Zn} \cdots \mathrm{NCCH}_{3}$ & 0.1951 & 0.4710 & 0.5617 \\
\hline $\mathrm{Cd} \cdots \mathrm{N}_{3}{ }^{-}$ & 0.2063 & 0.5841 & 0.8260 \\
\hline $\mathrm{Cd} \cdots \mathrm{NCCH}_{3}$ & 0.2157 & 0.2587 & 0.3941 \\
\hline
\end{tabular}

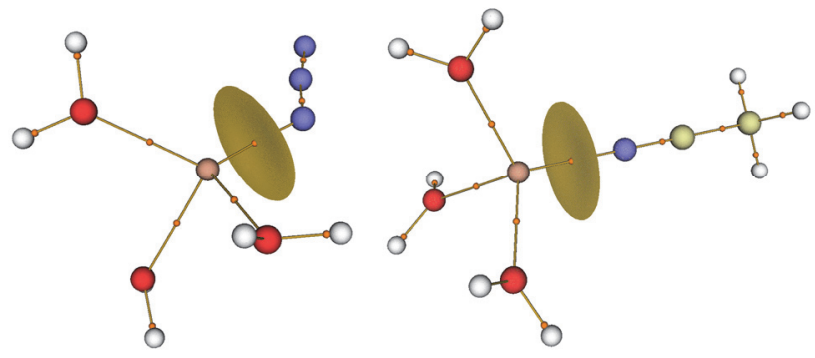

图 $2 \mathrm{Zn}^{2+}$ 离子活化的两种中间体的分子拓扑图

Figure 2 The molecular graph of the both $\mathrm{Zn}^{2+}$-activated intermediates

的配位原子间均存在一个键临界点, 表明金属锌离子和 配位原子之间有成键效应. 其它 10 种金属离子和 $\mathrm{Zn}^{2+}$ 离子相同, 与周围的四个配位原子均发生成键效应.

表 2 列出了 22 种中间体的 $\mathrm{M}-\mathrm{N}$ 配位键的键临界 点处的电子密度拓扑参数, 如电子密度 $(\rho(\mathrm{r}))$ 及其拉普 拉斯值 $\left(\nabla^{2} \rho(\mathrm{r})\right)$ 、电子动能密度 $(G(\mathrm{r}))$ 、电子势能密度 $(V(\mathrm{r}))$ 和电子总能量密度 $(H(\mathrm{r}))$. 计算结果表明 22 种中间 体的 $\mathrm{M}-\mathrm{N}$ 配位键的键临界点的 $\rho(\mathrm{r})$ 值均较小, $\mathrm{M}-$
$\mathrm{N}\left(\mathrm{N}_{3}{ }^{-}\right)$键的 $\rho(\mathrm{r})$ 在 $0.09 \sim 0.13$ a.u.之间, $\mathrm{M}-\mathrm{N}\left(\mathrm{CH}_{3} \mathrm{CN}\right)$ 键的 $\rho(\mathrm{r})$ 在 $0.05 \sim 0.09$ a.u.之间. 计算的 $\nabla^{2} \rho(\mathrm{r})>0$, 说明 $\mathrm{M}-\mathrm{N}$ 配位键的键临界点处的电荷是发散的. 如图 S2 的 拉普拉斯等值线图所示, $\mathrm{M}-\mathrm{N}$ 配位键的键临界点其位 于绿色实线区域，此处电荷是发散区域. 基于 $\mathrm{M}-\mathrm{N}$ 键 的键临界点的 $\rho(\mathrm{r})$ 和 $\nabla^{2} \rho(\mathrm{r})$ 值表明金属离子与配位原子 之间是典型的闭壳层相互作用 ${ }^{[20]}, \mathrm{M}-\mathrm{N}$ 配位键呈现离 子键特征. 此外, 计算的 $\mathrm{M}-\mathrm{N}$ 配位键的键临界点处的 $H(\mathrm{r})<0,|V(\mathrm{r})| / G(\mathrm{r})>1$, 表明 $\mathrm{M}-\mathrm{N}$ 键具有部分的共价

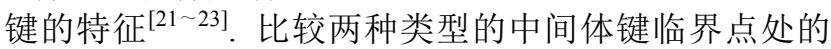
$\rho(\mathrm{r})$ 值大小, 可以看出 $\mathrm{M}-\mathrm{N}\left(\mathrm{N}_{3}{ }^{-}\right)$键的 $\rho(\mathrm{r})$ 比 $\mathrm{M}-$ $\mathrm{N}\left(\mathrm{CH}_{3} \mathrm{CN}\right)$ 键的大，其差值在 $0.02 \sim 0.05$ a.u. 之间，表明 $\mathrm{M}-\mathrm{N}\left(\mathrm{N}_{3}{ }^{-}\right)$键的强度比 $\mathrm{M}-\mathrm{N}\left(\mathrm{CH}_{3} \mathrm{CN}\right)$ 键的大，在含金 属离子、叠氮酸根和有机腈的反应体系中, 更易于经配 位作用形成金属离子与叠氮酸根的中间体. 因此, 电子 密度拓扑分析也能够表明在反应体系中金属离子的作 用是活化叠氮酸根. 该结果与上述的键长和键级分析结 果一致.

表 2 中间体的 $\mathrm{M}-\mathrm{N}$ 键的键临界点处的电子密度拓扑参数

Table 2 The topological parameters at bond critical points of $\mathrm{M}-\mathrm{N}$ bonds for all intermediates obtained by AIM analysis

\begin{tabular}{|c|c|c|c|c|}
\hline BCPs & $\rho(\mathrm{r})$ & $\nabla^{2} \rho(\mathrm{r})$ & $|V(\mathrm{r})| / G(\mathrm{r})$ & $H(\mathrm{r})$ \\
\hline $\mathrm{Sc} \cdots \mathrm{N}_{3}^{-}$ & 0.0977 & 0.4202 & 1.1695 & -0.0214 \\
\hline $\mathrm{Sc} \cdots \mathrm{NCCH}_{3}$ & 0.0574 & 0.2474 & 1.0348 & -0.0022 \\
\hline $\mathrm{Ti} \cdots \mathrm{N}_{3}^{-}$ & 0.0987 & 0.4629 & 1.1591 & -0.0219 \\
\hline $\mathrm{Ti} \cdots \mathrm{NCCH}_{3}$ & 0.0641 & 0.3385 & 1.0286 & -0.0025 \\
\hline $\mathrm{V} \cdots \mathrm{N}_{3}^{-}$ & 0.1224 & 0.4672 & 1.2812 & -0.0457 \\
\hline $\mathrm{V} \cdots \mathrm{NCCH}_{3}$ & 0.0762 & 0.4218 & 1.0593 & -0.0066 \\
\hline $\mathrm{Cr} \cdots \mathrm{N}_{3}^{-}$ & 0.1035 & 0.4227 & 1.1926 & -0.0252 \\
\hline $\mathrm{Cr} \cdots \mathrm{NCCH}_{3}$ & 0.0813 & 0.4688 & 1.0306 & -0.0037 \\
\hline $\mathrm{Mn} \cdots \mathrm{N}_{3}^{-}$ & 0.1084 & 0.5518 & 1.1740 & -0.0291 \\
\hline $\mathrm{Mn} \cdots \mathrm{NCCH}_{3}$ & 0.0702 & 0.3512 & 1.0659 & -0.0062 \\
\hline $\mathrm{Fe} \cdots \mathrm{N}_{3}^{-}$ & 0.1187 & 0.6092 & 1.1730 & -0.0319 \\
\hline $\mathrm{Fe} \cdots \mathrm{NCCH}_{3}$ & 0.0764 & 0.4108 & 1.0547 & -0.0059 \\
\hline $\mathrm{Co} \cdots \mathrm{N}_{3}^{-}$ & 0.1167 & 0.5652 & 1.1690 & -0.0287 \\
\hline $\mathrm{Co} \cdots \mathrm{NCCH}_{3}$ & 0.0805 & 0.4638 & 1.0499 & -0.0061 \\
\hline $\mathrm{Ni} \cdots \mathrm{N}_{3}^{-}$ & 0.1172 & 0.6342 & 1.1311 & -0.0239 \\
\hline $\mathrm{Ni} \cdots \mathrm{NCCH}_{3}$ & 0.0831 & 0.4824 & 1.0398 & -0.0050 \\
\hline $\mathrm{Cu} \cdots \mathrm{N}_{3}^{-}$ & 0.1169 & 0.6734 & 1.1259 & -0.0242 \\
\hline $\mathrm{Cu} \cdots \mathrm{NCCH}_{3}$ & 0.0861 & 0.4654 & 1.0636 & -0.0079 \\
\hline $\mathrm{Zn} \cdots \mathrm{N}_{3}^{-}$ & 0.1144 & 0.5381 & 1.1622 & -0.0260 \\
\hline $\mathrm{Zn} \cdots \mathrm{NCCH}_{3}$ & 0.0878 & 0.4551 & 1.0820 & -0.0102 \\
\hline $\mathrm{Cd} \cdots \mathrm{N}_{3}^{-}$ & 0.0973 & 0.3709 & 1.1971 & -0.0228 \\
\hline $\mathrm{Cd} \cdots \mathrm{NCCH}_{3}$ & 0.0749 & 0.3380 & 1.1032 & -0.0097 \\
\hline
\end{tabular}




\section{3 相互作用能和势能面分析}

图 3 给出了计算的中间体中金属离子分别与叠氮酸 根和乙腈之间的相互作用能(键能)的计算值. 其中 $\mathrm{M}-$ $\mathrm{N}\left(\mathrm{N}_{3}{ }^{-}\right)$键的键能在 $-1082.74 \sim-1281.10 \mathrm{~kJ} / \mathrm{mol}$ 之间, 而 $\mathrm{M}-\mathrm{N}\left(\mathrm{CH}_{3} \mathrm{CN}\right)$ 键的键能在 $-222.84 \sim-325.93$ $\mathrm{kJ} / \mathrm{mol}$ 之间. 两者数值间的较大差距表明 $\mathrm{M}-\mathrm{N}\left(\mathrm{N}_{3}{ }^{-}\right)$键 的强度远远大于 $\mathrm{M}-\mathrm{N}\left(\mathrm{CH}_{3} \mathrm{CN}\right)$ 键的强度, 即金属离子 与叠氮酸根离子形成的中间体的稳定性远远地高于金 属离子与乙腈形成的中间体. 结果进一步表明金属离子 在该反应体系中起活化叠氮酸根的作用.

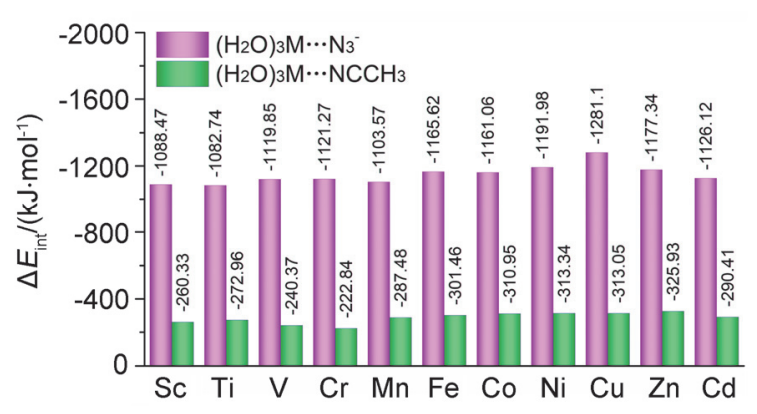

图 3 金属离子与乙腈和叠氮酸根阴离子间的相互作用能

Figure 3 The interaction energy between metal ions and acetonitrile or azide anion, respectively

另外, 为了解释 $\mathrm{M}-\mathrm{N}$ 键的强弱和中间体的稳定 性, 我们计算了中间体的 $\mathrm{M}-\mathrm{N}$ 键离解的势能面. 势能 面扫描结果表明 22 种中间体的 $\mathrm{M}-\mathrm{N}$ 键离解过程是一 个吸热的离解过程. 对同种金属离子形成的两种中间体 来说, 金属离子与叠氮酸根形成的中间体离解需要的能 量远远地高于金属离子与乙腈形成的中间体, 所以金属 离子与叠氮酸根形成的中间体在反应体系中更易于稳 定存在. 因此, 金属离子在反应体系中的作用是活化叠 氮酸根阴离子. 图 4 给出了部分中间体的 $\mathrm{M}-\mathrm{N}$ 键离解 的势能面, 其直观地呈现了 $\mathrm{M}-\mathrm{N}$ 键离解的特征和中间 体的稳定性的高低.

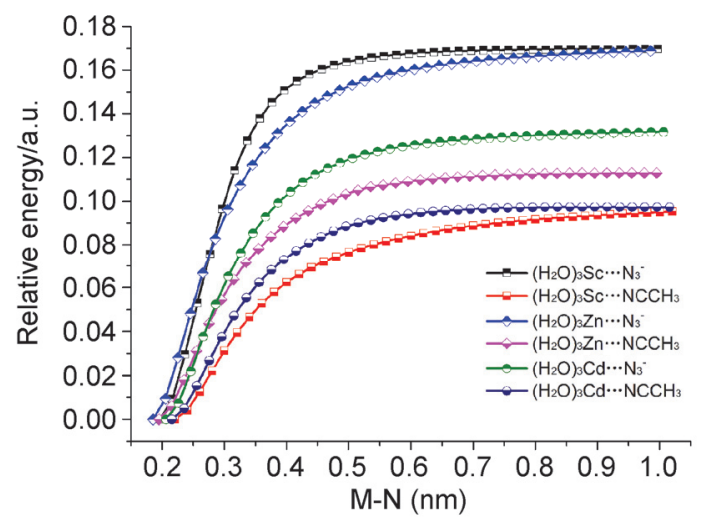

图 4 部分中间体的 $\mathrm{M}-\mathrm{N}$ 键离解的势能面图

Figure 4 The potential energy surface of $\mathrm{M}-\mathrm{N}$ bond dissociation for some intermediates

\section{4 静电势盆分析}

前述键长、键级、电子密度拓扑参数以及相互作用 能和势能面分析表明在反应体系中易于形成稳定的中 间体 $\left(\mathrm{H}_{2} \mathrm{O}\right)_{3} \mathrm{M} \cdots \mathrm{N}_{3}{ }^{-}$. 为了进一步探明金属离子催化下 有机腈和叠氮化钠反应的机理. 我们对 11 种中间体 $\left(\mathrm{H}_{2} \mathrm{O}\right)_{3} \mathrm{M} \cdots \mathrm{N}_{3}{ }^{-}$和乙腈分子表面活性位点进行了理论计 算与分析. 图 5 给出了 $\left(\mathrm{H}_{2} \mathrm{O}\right)_{3} \mathrm{Zn} \cdots \mathrm{N}_{3}{ }^{-}$和 $\mathrm{CH}_{3} \mathrm{CN}$ 分子静 电势盆分析的结果. 从中可以看出中间体中叠氮酸根未 配位的端基 $\mathrm{N}$ 原子盆中静电势值为正值(85.51 a.u.), 其 它中间体的介于 31.82 92.84 a.u.之间(图 S3), $\mathrm{CH}_{3} \mathrm{CN}$ 分子 $\mathrm{N}$ 原子盆中的静电势值为负值 $(-0.86$ a.u.). 从静 电势盆分析的结果可以看出，在亲核反应中，中间体中 叠氮酸根未配位的端基 $\mathrm{N}$ 原子是一个活性位置，易于受 到亲核试剂的进攻; 在亲电反应中, $\mathrm{CH}_{3} \mathrm{CN}$ 分子的 $\mathrm{N}$ 原 子将是亲电反应的活性位置, 易于受到亲电试剂的进 攻.

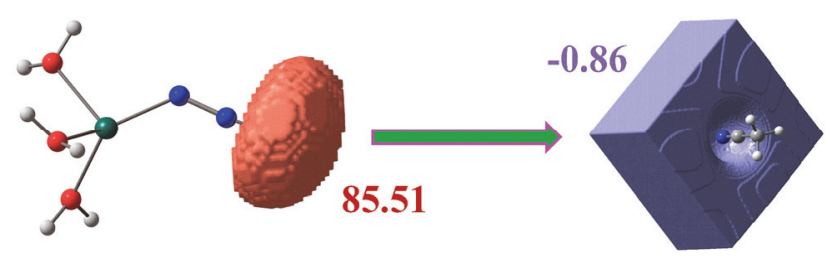

图 $5\left(\mathrm{H}_{2} \mathrm{O}\right)_{3} \mathrm{Zn} \cdots \mathrm{N}_{3}{ }^{-}$和 $\mathrm{CH}_{3} \mathrm{CN}$ 在相应的盆中的静电势值

Figure 5 The electrostatic potential values in the corresponding basins of $\left(\mathrm{H}_{2} \mathrm{O}\right)_{3} \mathrm{Zn} \cdots \mathrm{N}_{3}{ }^{-}$and $\mathrm{CH}_{3} \mathrm{CN}$

基于静电势盆分析，在反应体系中，中间体 $\left(\mathrm{H}_{2} \mathrm{O}\right)_{3} \mathrm{M} \cdots \mathrm{N}_{3}{ }^{-}$可以作为亲电试剂, 其未配位的端基 $\mathrm{N}$ 原子可以进攻乙腈的氰基 $\mathrm{N}$ 原子发生加成反应，随后发 生环加成反应原位形成四氮唑. 在水分子的辅助下，其 加成物也可能发生类似于里特反应 ${ }^{[24,25]}$ 生成酰胺类中 间体，其易于水解形成羧酸和胺. 我们称其为类里特反 应(Ritter-like reaction). 其可能的反应机理([2+3]环加 成反应和类里特反应)如图 6 所示. 我们预测的类里特 反应机理与目前理论和实验报道的金属离子催化下叠 氮酸根和有机腈原位生成四氮唑的反应机理完全不同. 我们基于提出的机理，已设计合成和捕获了首个七氮阴 离子 $\mathrm{N}_{7}{ }^{3-[19]}$. 这些结果表明我们提出反应机理是正确 的，把金属催化有机腈和叠氮酸根反应带入了一个新领 域.

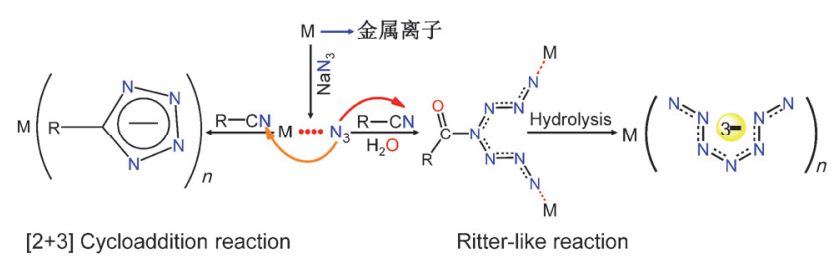

图 6 有机腈和叠氮酸根原位合成四唑或全氮阴离子的反应机理 Figure 6 The proposed mechanism of the formation of tetrazolyl compounds or polynitrogen anion via the in-situ reaction of nitrile with azide anion 


\section{5 中间体的实验捕获}

为了进一步验证前述理论计算结果, 我们利用水热 合成技术, 在同时含有叠氮化钠和 4-氟苯甲腈(4-FBN) 或 4'-(咪唑-1-基)苯甲腈(CPI)反应体系中, 在 $\mathrm{Cd}^{2+}$ 离子 的作用下, 成功捕获到了 $\mathrm{Cd}^{2+}$ 离子活化叠氮酸根的中 间体 1 和 2 , 而没有获得 $\mathrm{Cd}^{2+}$ 离子活化氰基基团的中间 体. 因此, 从实验结果也表明金属离子的作用是活化叠 氮酸根离子，与前述理论计算结果一致.

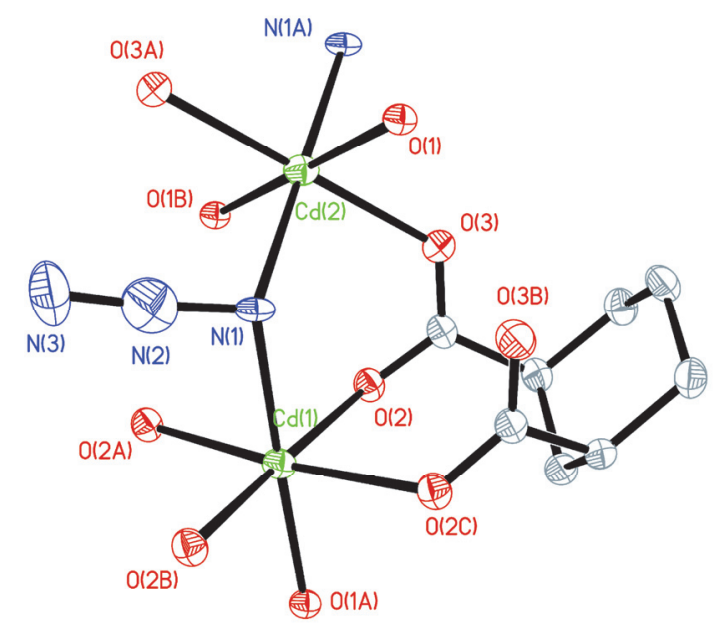

图 7 中间体 $\mathbf{1}$ 的中心 $\mathrm{Cd}^{2+}$ 离子的配位环境图, 为了清晰, 省略了所 有 $\mathrm{H}$ 原子

Figure 7 Coordination environments of the $\mathrm{Cd}^{2+}$ centers in $\mathbf{1}$ with the ellipsoids drawn at the $30 \%$ probability level, all hydrogen atoms are omitted for clarity.

中间体 1 的晶体数据和精修参数见表 S1. 1 的晶体 属于正交晶系, Pnma 空间群. 如图 7 所示, 其不对称单 元中两个独立的 $\mathrm{Cd}^{2+}$ 离子 $(\mathrm{Cd}(1)$ 和 $\mathrm{Cd}(2)$ 分别位于反映 面和倒反中心上, 其占有率均为 $1 / 2)$, 半个氢氧根, 半 个叠氮酸根, 半个 1,3 -环己二酸根 $\left(\mathrm{CHDA}^{2-}\right)$, 三者均位 于反映面上. $\mathrm{Cd}(1)$ 原子与两个 $\mathrm{CHDA}^{2-}$ 的四个羧基 $\mathrm{O}$ 原 子 $\left(\mathrm{Cd}-\mathrm{O} 0.2358(5)\right.$ 和 0.2334(4) nm), 一个 $\mu_{3}$ 桥联 $\mathrm{OH}^{-}$ 的一个 $\mathrm{O}$ 原子 $(\mathrm{Cd}-\mathrm{O} \quad 0.2191(6) \mathrm{nm})$ 以及一个 $\mu_{3}$ 桥联 $\mathrm{N}_{3}{ }^{-}$的一个 $\mathrm{N}$ 原子 $(\mathrm{Cd}-\mathrm{N} 0.2242(7) \mathrm{nm}$ )配位, 形成一个 畸变的八面体几何构型; 同样位于倒反中心上 $\mathrm{Cd}(2)$ 原 子也为畸变的八面体配位构型, 其赤道面上四个 $\mathrm{O}$ 原子 分别来自于两个 $\mathrm{CHDA}^{2-}$ 的两个羧基 $\mathrm{O}$ 原子和两个 $\mathrm{OH}^{-}$ 的两个 $\mathrm{O}$ 原子 $(\mathrm{Cd}-\mathrm{O}$ 0.2297(5)和 0.2268(4) nm), 两个 顶点上的 $\mathrm{N}$ 原子来自于两个不同的 $\mathrm{N}_{3}{ }^{-}(\mathrm{Cd}-\mathrm{N}$ $0.2353(5) \mathrm{nm})$. 与两个 $\mathrm{Cd}$ 原子相应的键角范围为 $82.69(19)^{\circ}$ 到 $180^{\circ}$ (表 S2). 每个 $\mu_{3}$ 桥联 $\mathrm{N}_{3}{ }^{-}$桥联邻近的三 个 $\mathrm{Cd}$ 原子形成沿 $b$ 轴方向伸展的一维无限阳离子 $\left[\mathrm{Cd}_{2}\left(\mathrm{~N}_{3}\right)\right]^{3+}$ 链, $\mu_{3}$ 桥联 $\mathrm{OH}^{-}$连接邻近的阳离子链形成沿 $b c$ 晶面无限伸展的二维阳离子 $\left[\mathrm{Cd}_{2}\left(\mathrm{~N}_{3}\right)(\mathrm{OH})\right]^{2+}$ 层, 且所 有的 $\mathrm{Cd}$ 原子位于同一个平面上. $\mathrm{CHDA}^{2-}$ 的经 $\mathrm{Cd}-\mathrm{O}$ 键 作用分布在二维阳离子层的两边形成中性的二维层(图
8). 邻近的二维层通过分子间弱相互作用以-AB-AB-模 式沿 $a$ 轴方向堆积成三维结构(图 9).

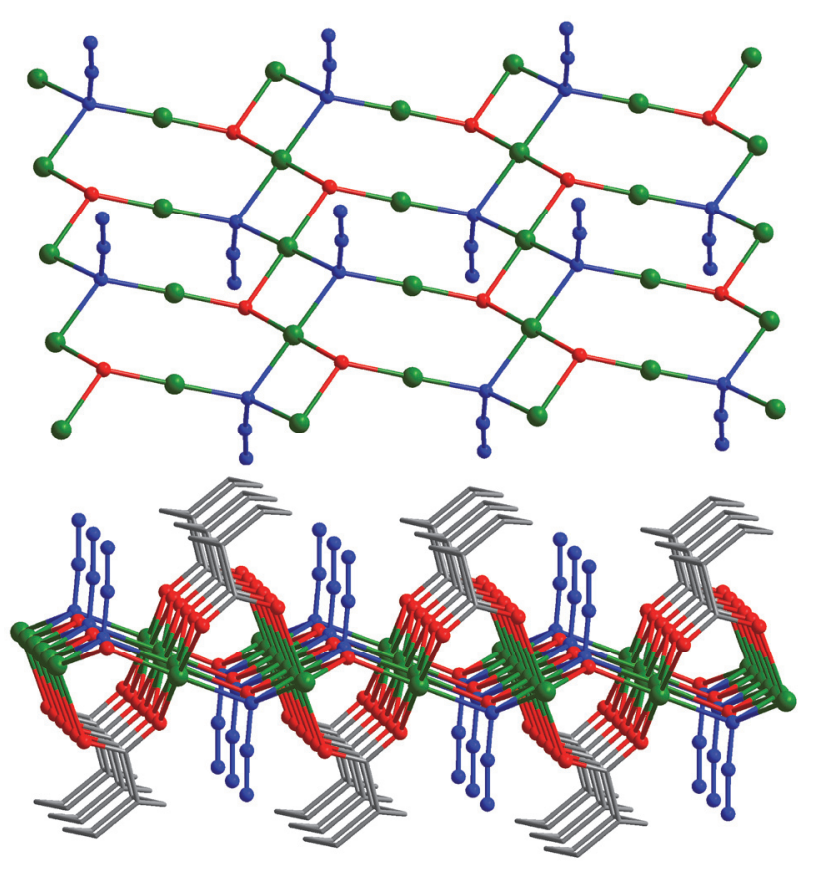

图 8 中间体 1 的二维层状结构图

Figure 8 A perspective view of the 2D layer in 1

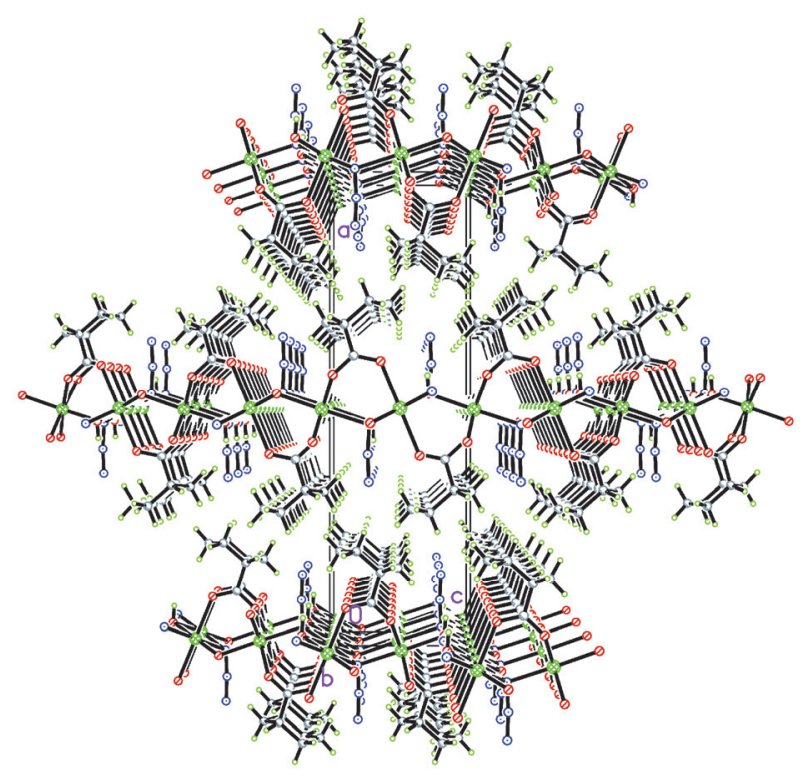

图 9 中间体 1 沿 $b$ 轴方向的晶体堆积图

Figure 9 Crystal packing diagram projected along the $b$-axis in $\mathbf{1}$

中间体 2 的晶体属于正交晶系, $P b c a$ 空间群，其不 对称单元含有一个中心 $\mathrm{Cd}^{2+}$ 离子, 一个叠氮酸根阴离 子和一个原位形成的 $\mathrm{IBA}^{-}$阴离子, 如图 10 所示. 中心 $\mathrm{Cd}^{2+}$ 离子采用三角双雉的配位构型, 其中赤道面上的三 个 $\mathrm{N}$ 原子分别来自于两个不同的 $\mathrm{N}_{3}{ }^{-}$酸根的 $\mathrm{N}$ 原子和一 个 $\mathrm{IBA}^{-}$阴离子咪唑基团上的 $\mathrm{N}$ 原子, 顶点上的两个 $\mathrm{O}$ 


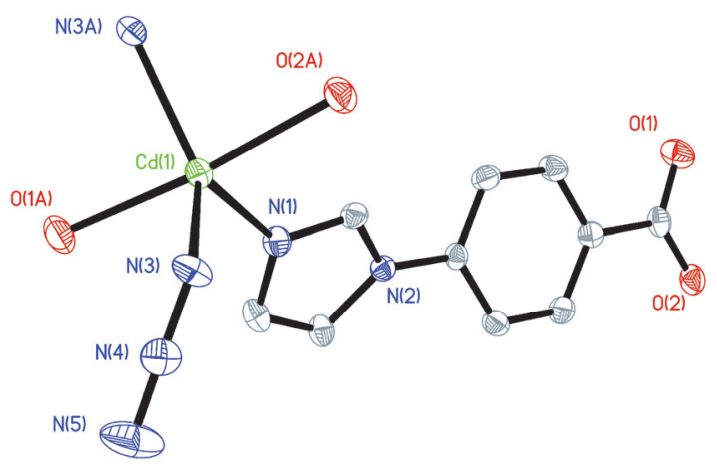

图 10 中间体 $\mathbf{2}$ 的中心 $\mathrm{Cd}^{2+}$ 离子的配位环境图, 为了清晰, 省略了所 有 $\mathrm{H}$ 原子

Figure 10 Coordination environments of the $\mathrm{Cd}^{2+}$ centers in $\mathbf{2}$ with the ellipsoids drawn at the $30 \%$ probability level, all hydrogen atoms are omitted for clarity

原子分别来自于两个 $\mathrm{IBA}^{-}$阴离子的羧基 $\mathrm{O}$ 原子, 其键 长和键角见表 $\mathrm{S} 3 . \mathrm{N}_{3}{ }^{-}$酸根采用 $\mu_{2}$ 桥联模式连接邻近的 两个中心 $\mathrm{Cd}^{2+}$ 离子形成沿 $b$ 轴方向无限伸展的一维 $\left[\mathrm{CdN}_{3}\right]^{+}$阳离子链, 如图 11 所示. 在链中, 两个邻近的 $\mathrm{Cd}^{2+}$ 离子间的距离为 $0.39157(0) \mathrm{nm}, \mathrm{Cd}-\mathrm{N}-\mathrm{Cd}$ 和 $\mathrm{N}-$ $\mathrm{Cd}-\mathrm{N}$ 键角分别为 $121.194(0)^{\circ}$ 和 $19.283(0)^{\circ}$. 邻近的 $\left[\mathrm{CdN}_{3}\right]^{+}$阳离子链经 $\mu_{3}$ 桥联模式的 $\mathrm{IBA}^{-}$阴离子通过配位 键作用形成三维的网状骨架结构, 如图 12 所示. 这个三 维骨架含有大的沿着 $b$ 轴方向的一维通道(近似为 $1.35394 \mathrm{~nm} \times 0.71830 \mathrm{~nm}$ ). 为了稳定该结构, 进一步被 一个相同结构三维结构贯穿, 形成二重贯穿的三维结 构, 如图 13 所示.

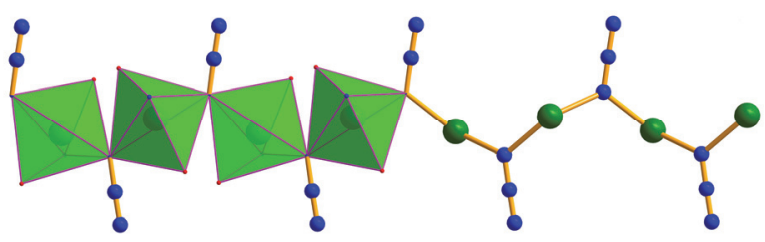

图 11 中间体 2 中一维沿 $b$ 轴伸展的 $\left[\mathrm{CdN}_{3}\right]^{+}$阳离子链

Figure $111 \mathrm{D}\left[\mathrm{CdN}_{3}\right]^{+}$cation chain extending along the $b$-axis in 2

\section{3 结论}

综上所述, 我们利用量子力学方法, 研究了金属离 子催化下有机腈和叠氮酸钠反应的机理. 基于优化的系 列过渡金属离子与叠氮酸根或乙腈分子形成的中间体 的结构和特性, 我们提出了金属离子在有机腈和叠氮化 钠反应中的作用是活化叠氮酸根而不是活化有机腈的 氧基基团的新见解. 同时基于分子表面活性位点分析和 我们已报道过的研究结果, 对金属离子催化下有机腈和 叠氮酸钠反应的机理给出了新的见解: (1)过渡金属离子 催化下有机腈和叠氮酸根反应合成四唑是以加成和环 合的分步机理进行的; (2)在水分子的辅助下，过渡金属 离子催化下有机腈和叠氮酸根可发生类里特反应合成 全氮阴离子. 此外, 实验上获得的金属中间体和全氮阴

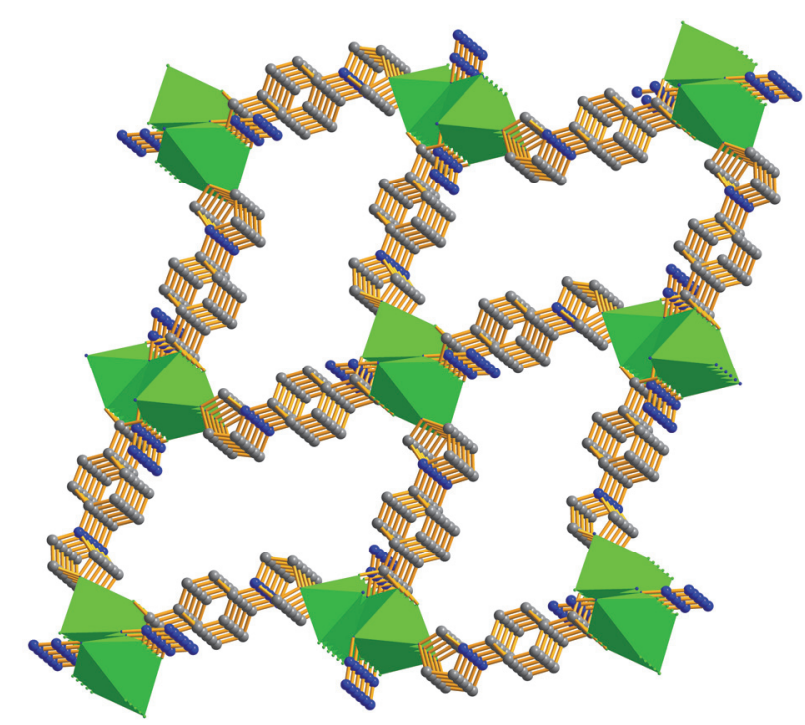

图 12 中间体 $\mathbf{2}$ 的三维结构图

Figure 12 Overview of 3D network of 2

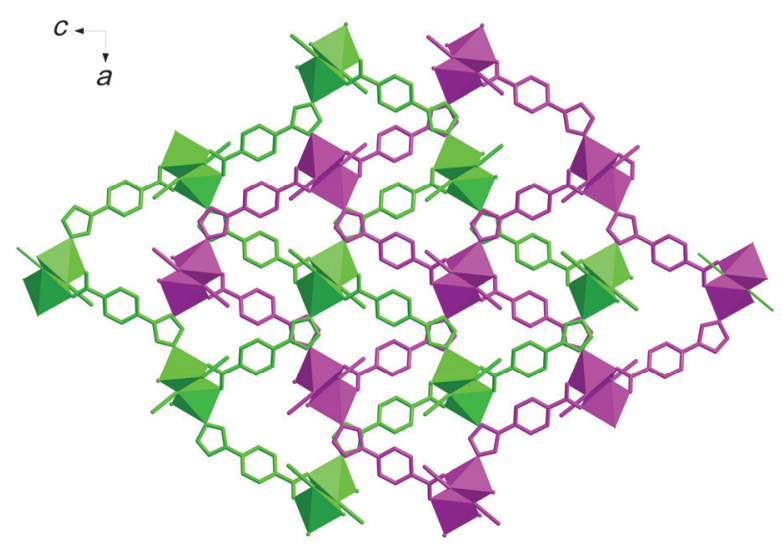

图 13 中间体 $\mathbf{2}$ 的 $3 \mathrm{D} \rightarrow 3 \mathrm{D}$ 的二重贯穿的三维结构图

Figure 13 Schematic representations of two-fold interpenetrating network of 2

离子 ${ }^{[19]}$ 也验证了我们提出的观点是正确的. 总之, 通过 理论计算和实验设计, 我们加深了对金属离子在有机腈 和叠氮酸根反应中的作用以及生成四氮唑的反应机理 的新认识，同时拓宽了金属离子催化下有机腈和叠氮酸 根反应合成全氮阴离子的新领域.

\section{4 实验部分}

\section{1 中间体 1 和 2 的合成}

$\mathrm{Cd}_{2}\left(\mu_{3}-\mathrm{N}_{3}\right)\left(\mu_{3}-\mathrm{OH}\right)\left(\mu_{5}-\mathrm{CHDA}\right) \quad(\mathbf{1})$ ：把 $\mathrm{Cd}\left(\mathrm{NO}_{3}\right)_{2}$ ・ $4 \mathrm{H}_{2} \mathrm{O},(0.0617 \mathrm{~g}, 0.2 \mathrm{mmol}), \mathrm{NaN}_{3}(0.0130 \mathrm{~g}, 0.2 \mathrm{mmol})$, 4-FBN (0.0242 g, $0.2 \mathrm{mmol}), \mathrm{H}_{2} \mathrm{CHDA}(0.0172 \mathrm{~g}, 0.1$ $\mathrm{mmol}), \mathrm{NaOH}(0.0080 \mathrm{~g}, 0.2 \mathrm{mmol})$ 和 $\mathrm{H}_{2} \mathrm{O}(12 \mathrm{~mL})$ 直接 混合，搅拌 $30 \mathrm{~min}$ 后，封入不锈钢反应釜的四氟乙烯内 胆内, 在 $170{ }^{\circ} \mathrm{C}$ 的烘箱内恒温 $3 \mathrm{~d}$ 后停止加热, 并自然 
冷却至室温, 移出产物, 过滤、洗涤、干燥, 得无色块状 晶体，产率 57\%, IR (KBr) v: 3602 (m), 2961 (m), 2936 (m), 2894 (w), 2089 (s), 1581 (s), 1459 (m), 1435 (m), 1416 (m), 1385 (s), 1354 (m), 1315 (w), 1280 (m), 1222 (w), $1106(\mathrm{w}), 1083(\mathrm{w}), 1005(\mathrm{w}), 947(\mathrm{w}), 881(\mathrm{w}), 846$ (w), $801(\mathrm{w}), 774(\mathrm{~m}), 731(\mathrm{~m}), 648(\mathrm{~m}), 616(\mathrm{~m}), 566(\mathrm{w})$, $579(\mathrm{w}), 505(\mathrm{w}), 450(\mathrm{w}), 408(\mathrm{w}) \mathrm{cm}^{-1}$. Anal. calcd for $\mathrm{C}_{8} \mathrm{H}_{11} \mathrm{~N}_{3} \mathrm{O}_{5} \mathrm{Cd}_{2}$ : C 21.16, H 2.44, N 9.25; found C 21.09, H 2.50, N 9.22.

$\mathrm{Cd}\left(\mu_{2}-\mathrm{N}_{3}\right)\left(\mu_{3}\right.$-IBA) (2): 中间体 2 的合成方法和 1 的 相同, 只是在反应中用 CPI $(0.0338 \mathrm{~g}, 0.2 \mathrm{mmol})$ 代替 4-FBN. 2 也为无色块状晶体, 产率 $51 \%$, IR (KBr) $v$ : 3135 (m), 3068 (w), 2926 (w), 2075 (s), 1605 (s), 1596 (s), 1541 (s), 1518 (s), 1495 (m), 1399 (s), 1387 (s), 1325 (m), 1305 (w), 1271 (m), 1244 (w), 1177 (w), 1123 (w), 1062 (m), $1012(\mathrm{w}), 963$ (w), $937(\mathrm{w}), 864(\mathrm{w}), 838(\mathrm{~m}), 779$ (m), $736(\mathrm{w}), 697(\mathrm{w}), 677(\mathrm{w}), 654(\mathrm{~m}), 551(\mathrm{w}), 515(\mathrm{w})$, 505 (w) $\mathrm{cm}^{-1}$. Anal. calcd for $\mathrm{C}_{10} \mathrm{H}_{7} \mathrm{~N}_{5} \mathrm{O}_{2} \mathrm{Cd}$ : C 35.16, $\mathrm{H}$ 2.07, N 20.50; found C 35.09, H 2.12, N 20.46.

\section{2 计算模型和方法}

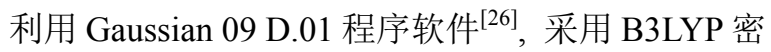
度泛函方法 ${ }^{[27,28]}$ ，计算中， $\mathrm{C} 、 \mathrm{H} 、 \mathrm{~N}$ 和 $\mathrm{O}$ 原子均采用

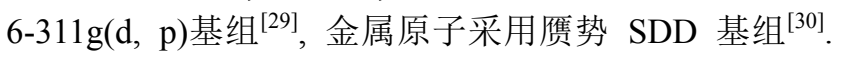
在上述计算级别下, 对系列含乙腈或叠氮酸根的金属离 子活化的中间体的基态结构进行了全优化和振动分析, 且振动分析计算无虚频. 计算模型如图 1 所示, 考虑到 金属离子的配位特点以及计算时间, 金属离子均采用四 配位模式, 配体为 3 个水分子和 1 个乙腈或 1 个叠氮酸 根, 其中金属离子均为 +2 价, 且为高自旋态. 依据 Bader 的 AIM 理论 ${ }^{[31]}$ 对获得的中间体进行了电子密度拓 扑分析. 金属离子和乙腈或叠氮酸根之间的相互作用能 为中间体能量和两个分子片段 $\left(\left[\mathrm{M}\left(\mathrm{H}_{2} \mathrm{O}\right)_{3}\right]^{2+}\right.$ 和乙腈分子 或叠氮酸根)的能量之差, 并利用 Boys 和 Bernard 提出 的方法 ${ }^{[32]}$ 考虑基组重叠效应. 此外, 利用 Multiwfn 程 序 ${ }^{[33]}$ 对金属离子活化的叠氮酸根的中间体和乙腈分子 进行了分子静电势盆分析.

\section{References}

[1] Demko, Z. P.; Sharpless, K. B. Angew. Chem., Int. Ed. 2002, 41, 2110 .

[2] Demko, Z. P.; Sharpless, K. B. Angew. Chem., Int. Ed. 2002, 41, 2113.

[3] Wu, T.; Yi, B. H.; Li, D. Inorg. Chem. 2005, 44, 4130.
[4] Ye, Q.; Wang, X. S.; Zhao, H.; Xiong, R. G. Chem. Soc. Rew. 2005, 34, 208.

[5] Chen, X. M.; Tong, M. L. Acc. Chem. Res. 2007, 40, 162.

[6] Li, J. R.; Tao, Y.; Yu, Q.; Bu, X. H. Chem. Commun. 2007, 1527.

[7] Zhao, H.; Qu, Z. R.; Ye, H. Y.; Xiong, R. G. Chem. Soc. Rev. 2008, $37,84$.

[8] Shang, J.; Zhang, J.; Cui, Y.; Zhang, T.; Shu, Y.; Yang, L. Acta Chim. Sinica 2010, 68, 233 (in Chinese). (尚静, 张建国, 崔燕, 张同来, 舒远杰, 杨利, 化学学报, 2010, 68, 233.)

[9] Cantillo, D.; Gutmann, B.; Oliver Kappe, C. J. Am. Chem. Soc. 2011, 133, 4465.

[10] Wang, S. H.; Zheng, F. K.; Wu, M. F.; Liu, Z. F.; Chen, J.; Guo, G. C.; Wu, A. Q. CrystEngComm 2013, 15, 2616.

[11] Li, X.; Cheng, L.; Fang, W.; Yang, G. Acta Chim. Sinica 2013, 71, 179 (in Chinese). (李新雄, 程琳, 方伟慧, 杨国昱, 化学学报, 2013, 71, 179.)

[12] Feng, Y.; Liu, X.; Duan, L.; Yang, Q.; Wei, Q.; Xie, G.; Chen, S.; Yang, X.; Gao, S. Dalton Trans. 2015, 44, 2333.

[13] Liu, X.; Gao, W.; Sun, P.; Su, Z.; Chen, S.; Wei, Q.; Xie, G.; Gao, S. Green Chem. 2015, 17, 831 .

[14] Xiong, R. G.; Xue, X.; Zhao, H.; You, X. Z.; Abrahams, B. F.; Xue, Z. Angew. Chem., Int. Ed. 2002, 41, 3800.

[15] Jin, T.; Kitahara, F. K.; Kamijo, S.; Yamamoto, Y. Chem. Asian J. 2008, 3, 1575.

[16] Himo, F.; Demko, Z. P.; Noodleman, L.; Sharpless, K. B. J. Am Chem. Soc. 2003, 125, 9983.

[17] Zhong, D. C.; Wen, Y. Q.; Deng, J. H.; Luo, X. Z.; Gong, Y. N.; Lu, T. B. Angew. Chem., Int. Ed. 2015, 54, 11795.

[18] Cantillo, D.; Gutmann, B.; Kappe, C. O. J. Am. Chem. Soc. 2011, $133,4465$.

[19] Huang, R. Y.; Zhang, C.; Yan, D.; Xiong, Z.; Xu, H.; Ren, X. M. $R S C$ Adv. 2018, 8, 39929.

[20] Bader, R. F. W.; Essén, H. J. Chem. Phys. 1984, 80, 1943.

[21] Crèmer, D.; Kraka, E. Croat. Chem. Acta 1984, 57, 1259.

[22] Cremer, D.; Kraka, E. Angew. Chem., Int. Ed. 1984, 23, 67.

[23] Espinosa, E.; Alkorta, I.; Elguero, J.; Molins, E. J. Chem. Phys. 2004, 387, 481 .

[24] Ritter, J. J.; Minieri, P. P. J. Am. Chem. Soc. 1948, 70, 4045.

[25] Ritter, J. J.; Kalish, J. J. Am. Chem. Soc. 1948, 70, 4048.

[26] Frisch, M. J.; Trucks, G. W.; Schlegel, H. B.; Scuseria, G. E.; Robb, M. A.; Cheeseman, J. R.; Scalmani, G.; Barone, V.; Mennucci, B.; Petersson, G. A.; Nakatsuji, H.; Caricato, M.; Li, X.; Hratchian, H.P.; Izmaylov, A. F.; Bloino, J.; Zheng, G.; Sonnenberg, J. L.; Hada, M.; Ehara, M.; Toyota, K.; Fukuda, R.; Hasegawa, J.; Ishida, M.; Nakajima, T.; Honda, Y.; Kitao, O.; Nakai, H.; Vreven, T.; Montgomery, J. A., Jr.; Peralta, J. E.; Ogliaro, F.; Bearpark, M.; Heyd, J. J.; Brothers, E.; Kudin, K. N.; Staroverov, V. N.; Keith, T.; Kobayashi, R.; Normand, J.; Raghavachari, K.; Rendell, A.; Burant, J. C.; Iyengar, S. S.; Tomasi, J.; Cossi, M.; Rega, N.; Millam, J. M.; Klene, M.; Knox, J. E.; Cross, J. B.; Bakken, V.; Adamo, C.; Jaramillo, J.; Gomperts, R.; Stratmann, R. E.; Yazyev, O.; Austin, A. J.; Cammi, R.; Pomelli, C.; Ochterski, J. W.; Martin, R. L.; Morokuma, K.; Zakrzewski, V. G.; Voth, G. A.; Salvador, P.; Dannenberg, J. J.; Dapprich, S.; Daniels, A. D.; Farkas, O.; Foresman, J. B.; Ortiz, J. V.; Cioslowski, J.; Fox, D. J. Gaussian 09, Revision D.01, Gaussian, Inc., Wallingford, CT, 2013.

[27] Becke, A. D. Phys. Rev. A 1998, 38, 3098.

[28] Lee, C.; Yang, W.; Parr, R. G. Phys. Rev. B 1988, 37, 785.

[29] Becke, A. D. J. Chem. Phys. 1993, 98, 5648.

[30] Andrae, D.; Häuerman, U.; Dolg, M.; Stoll, H.; Preu, H. Theor. Chim. Acta 1990, 77, 123 .

[31] Bader, R. F. W. Atoms in Molecules, A Quantum Theory, Oxford University Press, Oxford, 1990.

[32] Boys, S. F.; Bernardi, F. Mol. Phys. 1970, 19, 553.

[33] Lu, T.; Chen, F. W. J. Comp. Chem. 2012, 33, 580. 